Article

\title{
Two-Stage Active and Reactive Power Coordinated Optimal Dispatch for Active Distribution Network Considering Load Flexibility
}

\author{
Yu Zhang ${ }^{1}$, Xiaohui Song ${ }^{1}$, Yong $\mathrm{Li}^{2, *} \mathbb{0}$, Zilong Zeng ${ }^{2, *}$, Chenchen Yong ${ }^{2}$, Denis Sidorov ${ }^{3,4} \mathbb{C}$ \\ and Xia $\mathbf{L v}^{5}$ \\ 1 China Electric Power Research Institute Co., Ltd., Beijing 100192, China; \\ zhangyu2010@epri.sgcc.com.cn (Y.Z.); songxh@epri.sgcc.com.cn (X.S.) \\ 2 College of Electrical and Information Engineering, Hunan University, Changsha 410082, China; \\ ycc1102@hnu.edu.cn \\ 3 Energy Systems Institute, Russian Academy of Sciences, 664033 Irkutsk, Russia; dsidorov@isem.irk.ru \\ 4 Industrial Maths Lab of Irkutsk National Research Technical University, 664033 Irkutsk, Russia \\ 5 Zhuhai Powint Electric Co. Ltd., Zhuhai 519085, China; lvxia678@163.com \\ * Correspondence: yongli@hnu.edu.cn (Y.L.); zengzilong@hnu.edu.cn (Z.Z.)
}

Received: 5 August 2020; Accepted: 9 November 2020; Published: 13 November 2020

\begin{abstract}
A high proportion of renewable energy connected to the power grid has caused power quality problems. Voltage-sensitive loads are extremely susceptible to voltage fluctuations, causing power system safety issues and economic losses. Considering the uncertainty factor and the time-varying characteristic, a linearized random ZIP model (constant impedance (Z), constant current (I), and constant power $(\mathrm{P})$ ) with time-varying characteristics was proposed. In order to improve the voltage quality of the voltage-sensitive loads in the day-here stage in an active distribution network (ADN), a linearized two-stage active and reactive power coordinated stochastic optimization model was established. The day-ahead active and reactive power coordination optimization was to smooth the large voltage fluctuation and develop a reserve plan to eliminate the unbalanced power caused by the prediction error in the day-here optimization. In the day-here real-time redispatch, the voltage was further improved by the continuous reactive power compensation device. Finally, the simulation results on the IEEE-33 bus system showed that the control strategy could better eliminate the unbalanced power caused by the prediction error and obviously improve the voltage of sensitive loads in the real-time stage on the premise of maintaining economic optimality.
\end{abstract}

Keywords: highly sensitive load; economic dispatch; stochastic planning; multiple time scales; voltage control

\section{Introduction}

The rapid development worldwide has increased the demand for power construction. With the gradual expansion of the energy crisis and severe environmental problems, sustainable development has become the main goal of future power grid construction. The application of renewable energy source (RES) and distributed energy has developed rapidly. However, with the large amount of RESs connected to the grid, its volatilities and uncertainties have brought huge challenges to the operation and regulation to the active distribution network (ADN) [1]. Optimization and dispatch of the traditional ADN is carried out according to the method of generation load tracking. When a large proportion of RESs are connected, the ADN needs to reserve a large amount of power to eliminate the effect of these uncertainties, which not only reduces the efficiency of generators, but also increases the dispatching cost of the ADN [2,3]. Therefore, it is necessary to utilize the initiative and flexibility of 
the flexible load on the demand side. The economic cost of the distribution network for an ADN is reduced by optimizing the dynamic active and reactive power.

RESs will cause a certain degree of voltage fluctuations after being connected to the grid. The penetration rate of RESs is greater, and the voltage fluctuations within a short period are greater. Most of the equipment and electrical loads in the semiconductor manufacturing, information, computer, or electronic communications industries are highly sensitive loads [4]. The operating state of many industries like electronic communications, adjustable speed motors, direct current (DC) motor drives, alternating current $(\mathrm{AC})$ contactors, programmable logic, and other equipment are affected by voltage fluctuations, which cause huge economic losses $[5,6]$. These highly sensitive loads have higher requirements for voltage quality and reactive power compensation $[7,8]$. Different types of highly sensitive loads do not have the same reference to sensitivity in power quality problems, which have great uncertain characteristics. Through the above analysis, it is necessary to comprehensively consider the flexibility of flexible loads and the loss cost of highly sensitive loads, and set up a reasonable active and reactive power optimal dispatch method to ensure the stability and the reasonable economic cost of an ADN.

There have been some studies on the optimal dispatch and voltage control of a distribution network. In [9], the division of an autonomous region active power control strategy was proposed to improve the voltage control ability of an ADN and make it work normally under large power change. In [10], a chance constrained programming (CCP) model based on the k-means clustering method was proposed considering the uncertainty of a multi-stage planning load and wind power in the ADN. In [11], a hierarchical coordination optimization model considering prediction error to eliminate risks based on chance-constrained programming was proposed to minimize the cost of exchange companies and achieve the best trend. In [12], the stochastic optimal Benders decomposition method considering the uncertainties of flexible load and intermittent energy in the distribution network was established for achieving optimal operation and efficient utilization of intermittent energy in the worst case. In [13], an optimization planning model considering network loss and system reliability was established based on the Pareto optimization method, which realizes the multi-objective optimization of ADN. In [14], a new distributed voltage control strategy based on a multi-agent system platform was proposed that could realize the overall network voltage management and maximize the distributed generation. In [15], multi-objective optimization algorithm models were applied to realize the economic and optimization of the distribution system to reduce the load loss and the cost of network investment. The above literature did not consider the impact of highly sensitive loads in an ADN, nor did it perform real-time regulation and dispatch based on the flexibility and randomness of the load. Therefore, on the basis of ensuring the stability of the highly sensitive load, two-stage optimization and dispatching of an ADN was carried out to optimize the economic cost of the distribution network.

In this paper, a linearized random voltage-sensitive load model was proposed to contain the time characteristics and the uncertainties of the load. A linearized two-stage active and reactive power stochastic coordinated optimization model was established to reduce the influence of voltage fluctuations and uncertainties on these sensitive loads. The day-ahead active and reactive power coordination optimization was to smooth large voltage fluctuations and develop a reserve plan to eliminate the unbalanced power caused by the prediction error in the day-here optimization. In the day-here real-time redispatch, the voltage was further improved by the continuous reactive power compensation devices. Finally, the simulation on the IEEE-33 bus system showed that the proposed coordinated optimal dispatch could effectively improve the voltage level of the sensitive load in the closed-loop method and minimize the operation cost of an ADN.

The rest of this paper is organized as follows. Section 2 presents the linearized random ZIP model (constant impedance $(\mathrm{Z})$, constant current $(\mathrm{I})$, and constant power $(\mathrm{P})$ ) considering the time-varying characteristics. In Section 3, the two-stage active and reactive power coordinated optimal dispatch for ADN are given. Simulation results in a modified IEEE 33-bus system are presented in Section 4. Finally, Section 5 presents the conclusion. 


\section{Linearized Random ZIP Model Considering Time-Varying Characteristics}

The load in the power system is not all constant, and most of the load will be affected by the operating state of the system, especially the voltage of the bus [16]. In this paper, we mainly considered the static characteristics of the load, which can be described by the ZIP model [17]. The ZIP model divides the entire load into three parts including the constant impedance, constant power, and constant current, the traditional ZIP model cannot describe the random characteristics and the time-varying characteristics [18,19]. Therefore, an improved ZIP model was proposed, which is shown as follows:

$$
\begin{aligned}
P_{m, i, t} & =\left(a_{P, t}\left(\frac{v_{i, t}}{v_{0}}\right)^{2}+b_{P, t}\left(\frac{v_{i, t}}{v_{o}}\right)+c_{P, t}\right)\left(P_{m, i, t}^{*}+\delta_{P, m, i, t}\right) \\
Q_{m, i, t} & =\left(a_{Q, t}\left(\frac{v_{i, t}}{v_{0}}\right)^{2}+b_{Q, t}\left(\frac{v_{i, t}}{v_{0}}\right)+c_{Q, t}\right)\left(Q_{m, i, t}^{*}+\delta_{Q, m, i, t}\right)
\end{aligned}
$$

where $a_{P, t}, b_{P, t}, c_{P, t}, a_{Q, t}, b_{Q, t}, c_{Q, t}$ represent the ratio of constant impedance $a(\mathrm{CI})$, constant power $b(\mathrm{CP})$, and constant current $c(C C)$ in this load at time $t$, respectively, and $a_{P, t}+b_{P, t}+c_{P, t}=a_{Q, t}+b_{Q, t}+c_{Q, t}=1$. This paper set $a_{P, t}=a_{Q, t}, b_{P, t}=b_{Q, t}, c_{P, t}=c_{Q, t} ; P_{m, i, t}^{*}$ and $Q_{m, i, t}^{*}$ are the predicted active value and reactive value of the load at bus $i$ in time $t$, respectively; $v_{i, t}$ is the voltage of bus $i$ in time $t ; v_{0}$ is the unit value of power system voltage, $v_{0}=1.0$ p.u.; $P_{m, i, t}$ and $Q_{m, i, t}$ are the actual active/reactive power of load at bus $i$ in time $t$, respectively; and $\delta_{P, m, i, t}$ and $\delta_{Q, m, i, t}$ are the uncertainty of the active/reactive power demand at bus $i$ in time $t$.

Consider that there is a square term of voltage in Equations (1) and (2), which builds a nonlinear non-convex model. In the actual operation of the power system, the voltage is very close to the standard unit value 1.00 p.u. Therefore, this paper carried out Taylor's first-order expansion on the square term of voltage at $v_{o}=1.00$ p.u. and ignored the higher-order terms. Obtain $v_{i}{ }^{2}=2 v_{i}-1$ and substitute it in Equations (1) and (2) as:

$$
\begin{gathered}
P_{m, i, t}=\left(\left(2 a_{P, t}+b_{P, t}\right) v_{i, t}+\left(c_{P, t}-a_{P, t}\right)\right)\left(P_{m, i, t}^{*}+\delta_{P, m, i, t}\right) \\
Q_{m, i, t}=\left(\left(2 a_{Q, t}+b_{Q, t}\right) v_{i, t}+\left(c_{Q, t}-a_{Q, t}\right)\right)\left(Q_{m, i, t}^{*}+\delta_{Q, m, i, t}\right)
\end{gathered}
$$

Compared with the traditional ZIP model, the proposed improved ZIP model can well express the static voltage, time-varying, and random characteristics of sensitive loads.

\section{Two-Stage Active and Reactive Power Coordinated Optimal Dispatch for an Active Distribution Network (ADN)}

Considering that day-ahead scheduling formulates instructions based on predicted values, these predicted values have a certain degree error. Therefore, it is necessary to introduce the active and reactive power redispatch in the day-here optimization for eliminate the predicted error. Due to the predicted error, pursuing voltage quality in the day-ahead scheduling stage will increase the control cost and reduce the robustness of the power system. Therefore, this paper proposed a two-stage active and reactive power coordinated control strategy for ADN, which takes the efficient and reliable power supply of highly sensitive loads as the goal. The objective function of the proposed model was the sum of the day-ahead scheduling cost and the expected value in the real-time scheduling cost. The OLTC (on-load tap changer) was used to smooth out large voltage fluctuations for sensitive loads in the day-ahead scheduling stage [20]. In the real-time optimization stage, the continuous reactive power control devices distributed in the system were used to further control the bus voltage [21].

\subsection{Objective Function}

From the perspective of power grid operation, the dispatching of an ADN should pursue the maximization of social value and minimize the operating costs. Load utility represents the benefits brought to users by demand-side electricity consumption. Non-flexible loads do not have load utility 
because they do not have elastic utility. Only the flexible load is considered to provide backup increased load utility costs. The two-stage active and reactive power coordination optimization model proposed in this paper was based on the minimum operating cost of an ADN, which includes day-ahead scheduling costs and the expected value of the real-time scheduling costs. The day-ahead total costs include power purchase costs from the main system, fuel costs, and backup costs of the DG (distribution generator) power generation, backup costs of the flexible load, and day-ahead power network loss costs. The spinning reserve of flexible load and the DG determined by day-ahead are mainly used to balance the power imbalance caused by the prediction error in the real-time scheduling. Real-time scheduling costs mainly include the expected cost of the sum power network loss costs and highly sensitive load loss costs [22,23].

The objective function is shown in Equation (5):

$$
\begin{gathered}
\min _{D A}+F_{D I} \\
F_{D A}=\sum_{t \in N_{t}}\left\{\sum _ { i \in \Omega _ { i } } \left[\sum_{g \in \Omega_{g, i}}\left(C_{g} P_{g, i, t}+C_{g}^{B} \alpha_{g, i, t}^{g n}+C_{g}^{S} \beta_{g, i, t}^{g n}+C_{g}^{U} P_{g, i, t}^{U}+C_{g}^{D} P_{g, i, t}^{D}\right)+\right.\right. \\
\left.\left.\sum_{l \in \Omega_{l, i}}\left(C_{l, t}^{U} P_{l, i, t}^{U}+C_{l, t}^{D} P_{l, i, t}^{D}\right)+\sum_{e \in \Omega_{e, i}}\left(C_{e, p} P_{e, i, t}+C_{e, q} Q_{e, i, t}\right)\right]+\sum_{i j \in \Omega_{l i n e}}\left(C_{l o s s}^{A} P_{l o s s, i j, t}^{A}\right)\right\} \\
F_{D I}=\sum_{r \in R} \pi_{r} \sum_{t}\left(C_{l o s s}^{I} P_{l o s s, i j, t, r}^{I}+\sum_{i \in \Omega_{i}} \sum_{m \in \Omega_{m, i}} C_{m, t} P_{m, i, t, r}\right) \\
P_{l o s s, i j, t}^{A}=g_{i j}\left(\sum_{y=1}^{Y} m_{i j, y} \theta_{i j, y, t}\right) \\
P_{l o s s, i j, t, r}^{I}=g_{i j}\left(\sum_{y=1}^{Y} m_{i j, y} \theta_{i j, y, t, r}\right) \\
\Delta P_{m, i, t, r}=P_{m, i, t, r}^{+}+P_{m, i, t, r}^{-} \\
P_{m, i, t, r}^{+}-P_{m, i, t, r}^{-}=P_{m, m a x, r}-P_{m, i, t, r} \\
P_{m, i, t, r}^{+} \geq 0 \\
P_{m, i t, r}^{-} \geq 0
\end{gathered}
$$

In the above equations, $t, i, g, e, l, m, r$ represent the indices of time, bus, generator, main grid, flexible load, sensitive load, and the random scene, respectively; $N_{t}$ represents the set of time period; $\Omega_{i}$ represents the set of bus; $\Omega_{g, i}$ represents the set of generator at bus $i ; \Omega_{l, i}$ represents the set of the flexible load at bus $i ; \Omega_{e, i}$ represents the set of the main grid at bus $i$; $\Omega_{\text {line }}$ represents the set of line; and $\Omega_{m, i}$ represents the set of the sensitive load at bus $i$.

Day-ahead dispatch cost $F_{D A}$ includes four parts: power generation and reserve cost, flexible load reserve cost, power purchase cost, and day-ahead power network loss cost. $C_{g}, C_{g}^{U}$, and $C_{g}^{D}$, respectively, represent the marginal cost, up/down spinning reserve cost of power generator $g ; C_{g}^{B}$ and $C_{g}^{S}$ represent the startup and shutdown costs of power generator $g$, respectively; $\alpha_{g, i, t}^{g n}$ and $\beta_{g, i, t}^{g n}$ are the startup and shutdown binary integer commands of power generator $g$ at bus $i$ in time period $t$, respectively; $P_{g, i, t}$ is the output power of power generator $g$ at bus $i$ in time period $t ; P_{g, i, t}^{U}$ and $P_{g, i, t}^{D}$ are the up- and down-reserved capacity of power generator $g$ at bus $i$ in time period $t$, respectively; $C_{l, t}^{U}$ and $C_{l, t}^{D}$ are the up/down spinning reserve cost of the flexible load $l$ in time period $t$, respectively; $P_{l, i, t}^{U, t}$ and $P_{l, i, t}^{D}$ are the up/down spinning reserve of the flexible load $l$ at bus $i$ in time period $t$, respectively; $P_{e, i, t}^{l, t}$ and $Q_{e, i, t}$ are the active and reactive power purchased from the main grid at bus $i$ in time period $t$, respectively; $C_{e, p}$ and $C_{e, q}$ are the unit purchased price of active power and reactive power, respectively; and $C_{\text {loss }}^{A}$ and $P_{\text {loss }, i j, t}^{A}$ are the unit electricity price of day-ahead power network loss and the day-ahead power network loss of line $i j$ in time period $t$.

The expected real-time cost $F_{D I}$ is the product of the real-time cost of each scene and the scene probability $\pi_{r} ; C_{\text {loss }}^{I}$ and $P_{\text {loss }, i j, t, r}^{I}$ are the unit electricity price of real-time power network loss and the 
real-time power network loss of line $i j$ in time period $t$ under the scene $r$, respectively; $R$ is a set of real-time scenes. The set in this paper was $5 . C_{m, t}$ is the unit price of the highly sensitive load loss; and $\Delta P_{m, i, t, r}$ is the real-time loss of a highly sensitive load in the time period $t$ under the scene $r$.

In Equations (8) and (9), $g_{i j}$ is the conductance between bus $i$ and bus $j$ in the time period $t ; m_{i j, y}$ is the linearized parameter that will be explained in detail later; $Y$ variables are those needed to model the domain restriction of the function to the positive orthant. $\theta_{i j, y, t}$ and $\theta_{i j, y, r, t}$ are the segmented value of the voltage phase angle difference between the two ends of the bus $i$ and bus $j$ in the time period $t$ in the day-head optimization or in the scenes $r$ of the day-here optimization, respectively. In Equations (10)-(13), two slack variables $P_{m, i, t, r}^{+}$and $P_{m, i, t, r}^{-}$are introduced to obtain the change of highly sensitive load loss. $P_{m, m a x, r}$ and $P_{m, i, t, r}$ are the active power values of the high-sensitive load when the voltage is close to $1.0 \mathrm{p} . \mathrm{u}$ and the actual value of the high-sensitive load in time period $t$ under the scene $r$.

\subsubsection{Constraints for the Day-Ahead Dispatch}

1. Constraints for the generator

Equations (15) and (16) are the minimum startup time constraint of the generators; Equations (17) and (18) are the minimum shutdown time constraint of the generators; Equations (19) and (20) are the up- and down-ramp constraints of the generators; Equations (21) and (22) are the output active capacity constraints of the generators; Equation (23) is the reactive power constraint of the generators; and Equations (24) and (25) are the range of the upper and lower reserve of the generators.

$$
\begin{gathered}
\alpha_{g, i, t}^{g n}-\beta_{g, i, t}^{g n}=u_{g, i, t}^{g n}-u_{g, i, t-1}^{g n} \\
\sum_{t}^{t+T_{g, i}^{o n, m i n}-1} u_{g, i, t}^{g n} \leq a_{g, i, t}^{g n} * T_{g, i}^{o n, m i n}\left(t \leq N_{t}-T_{g, i}^{o n, m i n}+1\right) \\
\sum_{t}^{N_{t}} u_{g, i, t}^{g n} \leq \alpha_{g, i, t}^{g n} *\left(N_{t}-T_{g, i}^{o n, m i n}+1\right)\left(t \geq N_{t}-T_{g, i}^{o n, m i n}+2\right) \\
t+T_{g, i}^{o f f, m i n}-1 \\
\sum_{t}\left(1-u_{g, i, t}^{g n}\right) \leq \beta_{g, i, t}^{g n} * T_{g, i}^{o f f, m i n}\left(t \leq N_{t}-T_{g, i}^{o f f, m i n}+1\right) \\
\sum_{t}^{N_{t}}\left(1-u_{g, i, t}^{g n} \leq \beta_{g, i, t}^{g n} *\left(N_{t}-T_{g, i}^{o f f, m i n}+1\right)\left(t \geq N_{t}-T_{g, i}^{o f f, m i n}+2\right)\right. \\
P_{g, i, t}+P_{g, i, t}^{U}-P_{g, i, t-1} \leq P_{g, i}^{g n, u p} u_{g, i, t}^{g n} \\
-P_{g, i, t}+P_{g, i, t}^{D}+P_{g, i, t-1} \leq P_{g, i}^{g n, d o w n} u_{g, i, t}^{g n} \\
P_{g, i, t}+P_{g, i, t}^{U} \leq u_{g, i, t}^{g n} P_{g, i, m a x}^{g n} \\
P_{g, i, t}-P_{g, i, t}^{D} \geq u_{g, i, t}^{g n} P_{g, i, m i n}^{g n} \\
u_{g, i, t}^{g n} Q_{g, i, m i n}^{g n} \leq Q_{g, i, t}^{g n} \leq u_{g, i, t}^{g n} Q_{g, i, m a x}^{g n} \\
0 \leq P_{g, i, t}^{U} \leq P_{g, i, t, m a x}^{U} \\
0 \leq P_{g, i, t}^{D} \leq P_{g, i, t, m a x}^{D}
\end{gathered}
$$

2. Constraints for the OLTC 
Equation (26) shows the voltage at both ends of the OLTC; Equation (27) shows the constraints of the tap; and Equation (28) shows the constraints of the allowable number of OLTC operations.

$$
\begin{gathered}
\mathrm{V}_{\mathrm{i} 0, \mathrm{t}}=\mathrm{V}_{\mathrm{i}, \mathrm{t}}\left(\mathrm{T}_{\mathrm{ij}, 1} \beta_{\mathrm{ij}, 1, \mathrm{t}}+\mathrm{T}_{\mathrm{ij}, 2} \beta_{\mathrm{ij}, 2, \mathrm{t}}+\cdots+\mathrm{T}_{\mathrm{ij}, \mathrm{H}} \beta_{\mathrm{ij}, \mathrm{H}, \mathrm{t}}\right) \\
\sum_{h=1}^{H} \beta_{i j, h, t}=1, \forall \beta_{i j, h, t} \in\{0,1\} \\
\sum_{t=1}^{23}\left(\left|\beta_{i j, 1}(t+1)-\beta_{i j, 1}(t)\right|+\cdots+\left|\beta_{i j, H}(t+1)-\beta_{i j, H}(t)\right|\right) \leq 2 N_{T}
\end{gathered}
$$

where $T_{i j}$ is the transformation ratio of OLTC, assuming that OLTC has an $H$ tap, the transformation ratio is $T_{i j} \in\left\{T_{i j, 1}, T_{i j, 2}, \cdots, T_{i j, H}\right\}$. In order to express the transformation ratio of the $h$ th tap, a binary variable $H$ is introduced at time $t$, which is $\beta_{i j, 1, t}, \beta_{i j, 2, t}, \cdots, \beta_{i j, H, t}$ [24]. $N_{T}$ is the maximum allowable number of OLTC operations, which was set to 4 in this paper.

3. Constraints for the flexible load

The flexible load participates in the day-ahead dispatch of the power system by providing a reserve. Constraints (33) and (34) show the amount of up/down spinning reserve capability of the flexible load [25].

$$
\begin{aligned}
& 0 \leq P_{l, i, t}^{U} \leq P_{l, i, t, \text { max }^{\prime}}^{U}, \forall i, t \\
& 0 \leq P_{l, i, t}^{D} \leq P_{l, i, t, \text { max }}^{D}, \forall i, t
\end{aligned}
$$

where $P_{l, i, t, \max }^{U}$ and $P_{l, i, t, \max }^{D}$ are the maximum schedulable reserve up and reserve down for the flexible load of the bus $i$ in the time period $t$, respectively. $P_{l, i, t}^{U}$ and $P_{l, i, t}^{D}$ represent the up/down spinning reserve at the bus $i$ in time, respectively.

4. Constraints for the SVC

Static var compensator (SVC) can not only output reactive power to the power grid, but also absorb reactive power from the power grid and realize continuous reactive power regulation. In order to simplify the model, the reactive power output of SVC connected to bus $i$ in the time period $t$ is expressed as $Q_{s, i, t}[24]$.

$$
Q_{s, i, \min } \leq Q_{s, i, t} \leq Q_{s, i, \max }
$$

where $Q_{s, i, \max }$ and $Q_{s, i, \min }$ are respectively the upper and lower limits of output reactive power of the SVC connected to bus $i$, which represent the out reactive power of SVC at bus $i$ in the time period $t$.

5. Constraints for the output active/reactive power of RES

The renewable energy considered in this paper was mainly wind power, which participates in the active and reactive power coordination optimization $[26,27]$. The output active power of the wind turbine is expressed as:

$$
P_{w, i, t}=\left\{\begin{array}{lc}
P_{w_{0}, i, t}\left(a+b v_{w, i, t}\right) & v_{w, i p}<v_{w, i, t}<v_{w_{o}} \\
P_{w_{0}, i, t} & v_{w_{0}}<v_{w, i, t}<v_{w, o p} \\
0 & v_{w, i, t} \leq v_{w, i p}, v_{w, i, t} \geq v_{w, o p}
\end{array}\right.
$$

where $P_{w, i, t}$ and $P_{w_{0}, i}$ are the output power of the wind turbine at the bus $i$ in the time period $t$ and rated power of the wind turbine at the bus $i$, respectively; $v_{w, i p}, v_{w, o p}$, and $v_{w_{0}}$ are the cut-in, cut-out, and rated wind speed of the wind turbine, respectively. $a$ and $b$ are the parameters related to the cut-in rated wind speed, which are fixed constants: $a=\frac{v_{w, i p}}{\left(v_{w, i p}-v_{w_{0}}\right)}, b=\frac{-1}{\left(v_{w, i p}-v_{w_{0}}\right)}$. 
Wind turbines are subject to the maximum apparent capacity of the inverter during operation, which is shown as follows:

$$
P_{w, i, t}^{2}+Q_{w, i, t}^{2} \leq S_{w, i, \max }^{2}
$$

where $S_{w, i, \max }$ is the maximum apparent capacity and $Q_{w, i, t}$ is the output reactive power of the wind turbines at bus $i$ in time $t$.

In order to simplify the calculation, linearize Equation (37) by using the hexagonal relaxation method:

$$
\begin{aligned}
-\sqrt{3}\left(P_{w, i, t}+S_{w, i, \max }\right) & \leq Q_{w, i, t} \leq-\sqrt{3}\left(P_{w, i, t}+S_{w, i, \max }\right) \\
-\frac{\sqrt{3}}{2} S_{w, i, t} & <Q_{w, i, t}<\frac{\sqrt{3}}{2} S_{w, i, t} \\
\sqrt{3}\left(P_{w, i, t}-S_{w, i, \max }\right)<Q_{w, i, t} & <\sqrt{3}\left(P_{w, i, t}+S_{w, i, \max }\right)
\end{aligned}
$$

6. The day-ahead power flow balance constraints

For the radial ADN, Equations (37) and (38) are the power flow constraints of the system:

$$
\begin{gathered}
\sum_{i j \in \Omega_{\text {line }, i}} P_{i j, t}=\sum_{i \in \Omega_{E, i}} P_{E, i, t}+\sum_{i \in \Omega_{g, i}} P_{g, i, t}+\sum_{i \in \Omega_{w}, i} P_{w, i, t}-\sum_{i \in \Omega_{l, i}} P_{l, i, t}-\sum_{i \in \Omega_{m, i}} P_{m, i, t} \\
\sum_{i j \in \Omega_{\text {line }, i}} Q_{i j, t}=\sum_{i \in \Omega_{E, i}} P_{E, i, t}+\sum_{i \in \Omega_{g, j}} Q_{g, i, t}+\sum_{i \in \Omega_{w, i}} Q_{w, i, t}+\sum_{i \in \Omega_{s, i}} Q_{s, i, t}-\sum_{i \in \Omega_{l, i}} Q_{l, i, t}-\sum_{i \in \Omega_{m, i}} Q_{m, i, t}
\end{gathered}
$$

In Equations (37) and (38), $N$ is the total number of buses in the system; $i$ and $j$ are the bus numbers; $\Omega_{\text {line, } i}$ is the set of lines connected with bus $i ; P_{i j, t}$ and $Q_{i j, t}$ are the total active/reactive power of the line $i j$ at time $t ; P_{w, i, t}, P_{l, i, t}, P_{m, i, t}$ are the active power of the wind turbine injected into bus $i$ in time $t$, the active power of the flexible load at bus $i$ in time $t$, and the active power of the sensitive load at bus $i$ in time $t$, respectively; $Q_{g, i, t}, Q_{w, i, t}, Q_{s, i, t}, Q_{l, i, t}$, and $Q_{m, i, t}$ are the reactive power of the generator injected into bus $i$ in time $t$, the reactive power of the wind turbine injected into bus $i$ in time $t$, the eactive power of the SVC injected into bus $i$ in time $t$, the reactive power of the flexible load at bus $i$ in time $t$, and the reactive power of the sensitive load at bus $i$ in time $t$, respectively.

$$
\begin{aligned}
P_{i j, t} & =g_{i j}\left(v_{i, t}-v_{j, t}\right)-b_{i j} \theta_{i j, t}+g_{i j, t} \\
Q_{i j, t} & =-b_{i j}\left(v_{i, t}-v_{j, t}\right)-g_{i j} \theta_{i j, t}-b_{i j, t} \frac{\theta_{i j, t}^{2}}{2}
\end{aligned}
$$

In Equations (39) and (40), $P_{i j, t}$ and $Q_{i j, t}$ are the active and reactive power of the line between bus $i$ and bus $j$ at time $t . \theta_{i j, t}$ is the voltage phase angle difference between bus $i$ and bus $j$ at time $t[28,29]$. In Equations (39) and (40), $\theta_{i j, t}^{2}$ is a non-linear variable, but it can be linearized by piecewise fitting linearity. As the piecewise fitting linearity was not the focus of this article, please refer to $[30,31]$ for details.

\subsubsection{Constraints for the Day-Here Dispatch}

1. Constraints for the active/reactive power redispatch of generators

Equations (41) and (42) are the real-time up and down reserve constraints of the generators; Equation (43) is the output of the power generator under the scene r; Equation (44) is the ramp constraint of the power generator in real-time stage; Equation (45) is the output reactive power constraints of generator under the scene $r$.

$$
0 \leq P_{g, i, t, r}^{U} \leq P_{g, i, t}^{U}, \forall i, t, r
$$




$$
\begin{gathered}
0 \leq P_{g, i, t, r}^{D} \leq P_{g, i, t}^{D} \forall i, t, r \\
P_{g, i, t, r}=P_{g, i, t}+P_{g, i, t, r}^{U}-P_{g, i, t, r^{\prime}}^{D}, \forall i, t, r \\
P_{g, i}^{g n, d o w n} \leq P_{g, i, t, r}-P_{g, i, t} \leq P_{g, i}^{g n, u p} \\
Q_{g, i, m i n}^{g n} \leq Q_{g, i, t, r}^{g n} \leq Q_{g, i, \max }^{g n}
\end{gathered}
$$

where $P_{g, i, t, r}^{U}$ and $P_{g, i, t, r}^{D}$ are respectively the upregulation and downregulation of the power generator under the scene $r ; P_{g, i, t, r}$ is the active output power of the power generator under the scene $r$; and $Q_{g, i t, r}^{g n}$ is the reactive output power of the power generator $g$ under scene $r$ in the time period $t$.

2. Constraints for the redispatch of flexible load

Constraints (46) and (47) are the upregulation and downregulation of the reserve constraint in the real-time stage of the flexible load. In Equation (48), $P_{l, i, t, r}$ is the active power output of the flexible load in the real-time stage under the scene $r$.

$$
\begin{gathered}
0 \leq P_{l, i, t, r}^{U} \leq P_{l, i, t^{\prime}}^{U} \forall i, t, r \\
0 \leq P_{l, i, t, r}^{D} \leq P_{l, i, t^{\prime}}^{D} \forall i, t, r \\
P_{l, i, t, r}=P_{l, i, t}+P_{l, i, t, r}^{U}-P_{l, i, t, r^{\prime}}^{D} \forall i, t, r
\end{gathered}
$$

where $P_{l, i, t, r}^{U}$ and $P_{l, i, t, r}^{D}$ represent the up/down spinning reserve deployed by the flexible load of the bus $i$ in the time period $t$ under the scene $r$.

In addition to the above Constraints (41)-(48), the day-here real-time optimization model also met the operational constraints of the SVC, RES, and power balance, which had a similar form to (31) and (34)-(38).

\section{Calculation Examples and Analysis}

The proposed two-stage active and reactive power coordination control strategy considering a random ZIP linearized model was tested in a modified IEEE 33-bus system. The specific modifications are as follows: (1) bus 33 was connected to a $0.5 \mathrm{MW}$ micro gas turbine; (2) bus 16 and bus 29 were connected to $0.9 \mathrm{MW}$ and $0.8 \mathrm{MW}$ wind turbines, respectively; (3) bus 6 and bus 18 were connected to 0.3 Mvar SVC controller devices, respectively; (4) the load at bus 14 and bus 26 were regarded as sensitive load buses; (5) the load at bus 7 and bus 24 are treated as the flexible loads with up to $30 \%$ of the load being able to be shed; and (6) the range of allowable voltage fluctuation was 0.930-1.070. The proposed model was coded in the GAMS environment using CPLEX 12.5. All experiments were implemented on a desktop computer with a processor clocked at $3.10 \mathrm{GHz}$ and $8 \mathrm{~GB}$ of RAM.

This paper mainly focused on the loss cost of highly sensitive loads. By comparing four cases that contained different reactive power regulation means, the effectiveness of the strategy proposed in this paper was illustrated: (1) Case 1: NO adjustment measure; (2) Case 2: RES adjustment measure; (3) Case 3: RES + SVC adjustment measure; and (4) Case 4: OLTC + RES + SVC adjustment measure.

The purpose of the day-ahead optimization was to provide sufficient reactive power reserve to reduce the effects of the uncertainty factors to the sensitive loads. Figure 1 shows the day-ahead voltage profile at the 14 bus and 26 bus under four cases. Case 4 was the best voltage regulation method compared to the other three cases. It can well regulate the voltage of different buses at different times. In Figure 1a, the overall voltage fluctuation ratio of 14 bus was $1.57 \%$ in Case $4,2.05 \%$ in Case $3,2.71 \%$ in Case 2, and $3.26 \%$ in Case 1, respectively. Case 4 can significantly reduce the overall voltage fluctuation compared with the other three cases. The minimum voltage of Case 1 at $16 \mathrm{~h}$ was 0.949 , which was very close to the lower limit of the voltage regulation range of 0.930 . When the reactive power compensation devices of RES, SVC, and OLTC were added, the voltage of the load was significantly improved. 
Especially after adding OLTC, the voltage increased most obviously, rising to 0.986 at $16 \mathrm{~h}$. In Figure $1 \mathrm{~b}$, the lowest voltage of the 26 bus was 0.965 in Case 1 at $16 \mathrm{~h}$. After the regulation of Case 4, the voltage increased to 0.996 at $16 \mathrm{~h}$. The overall voltage fluctuation ratio of bus 26 was $1.42 \%$ in Case $4 ; 2.01 \%$ in Case 3; $2.30 \%$ in Case 2, and 2.51\% in Case 1, respectively. The results showed that Case 4 had a good control effect on voltage at different times and overall voltage fluctuation of bus 26 . In the case of day-ahead scheduling, Case 4 could stabilize the overall voltage stability of different highly sensitive loads and also effectively adjust the voltage at different times.

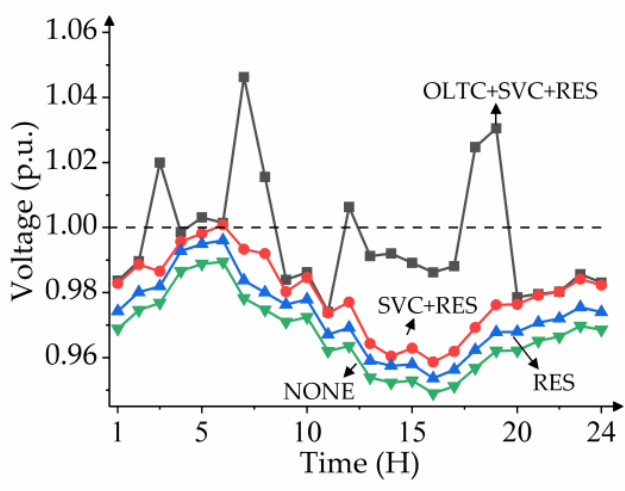

(a)

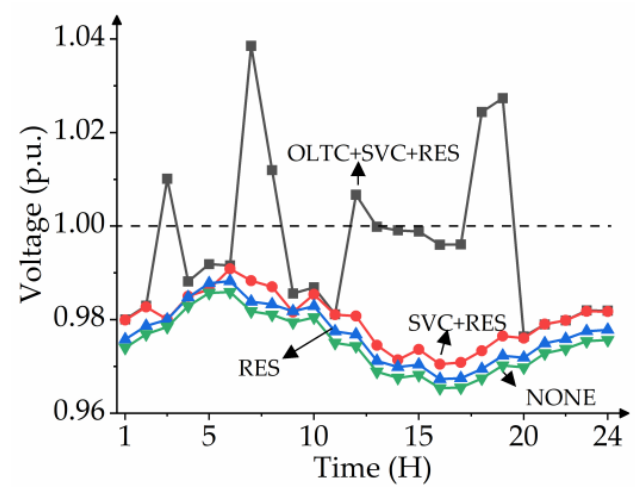

(b)

Figure 1. The result of the day-ahead optimization. (a) The day-ahead voltage profile of bus 14 .

(b) The day-ahead voltage profile of bus 26 .

In the day-here real-time redispatch, the voltage was further improved by the continuous reactive power compensation device. Figure 2 shows the real-time voltage changes under five different scenarios. In Figure 2a, the maximum voltage of Case 4 was 1.012 at $8 \mathrm{~h}$ and the average value of voltage at $8 \mathrm{~h}$ in the typical scene was close to the reference value, $1.00 \mathrm{p} . \mathrm{u}$, which means that the day-ahead optimization produced by Case 4 had relatively high robustness. Furthermore, the value of bus 14 at $7 \mathrm{~h}$ in the day-ahead stage was 1.046. While the related reactive control participated in the day-here stage, the voltage was further improved to 1.01. Furthermore, the overall voltage deviation ratio of bus 14 was $3.29 \%$ in Case 1, 2.78\% in Case 2, 1.63\% in Case 3, and $0.80 \%$ in Case 4, respectively. From Figure $2 \mathrm{a}$ at 10.00-20.00, the effect of these reactive power regulating devices was not obvious when the system load was heavy. For this situation, the voltage can be improved by making the day-ahead OLTC scheduling plan. Figure 3. shows the outlet voltage of the OLTC. Furthermore, the voltage profile of the ADN was obviously improved from Figure 4 after executing the day-ahead OLTC scheduling plan at 16h. A similar conclusion can be drawn from Figure $2 \mathrm{~b}$. In summary, Case 4 had the best voltage regulation effect in both the day-ahead and real-time stages.

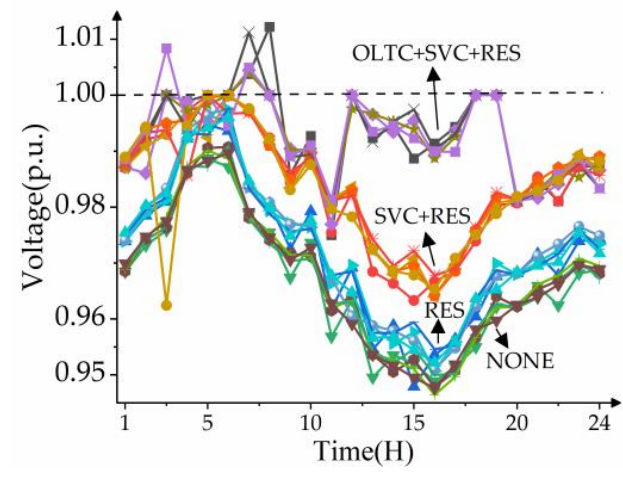

(a)

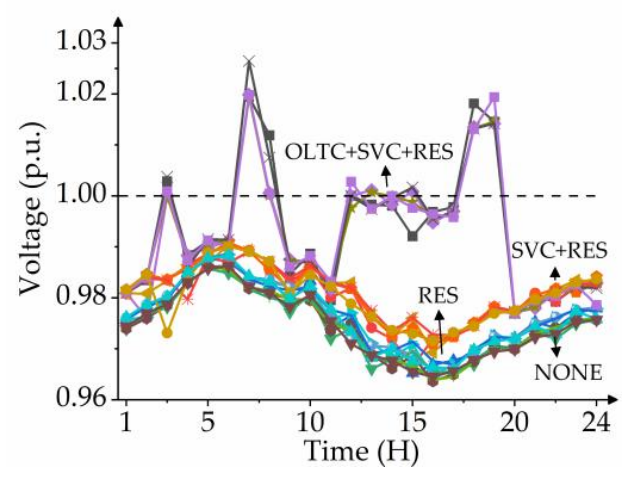

(b)

Figure 2. The result of the real-time dispatching in different typical scenes. (a) The day-here voltage profile of bus 14. (b) The day-here voltage profile of bus 26 . 


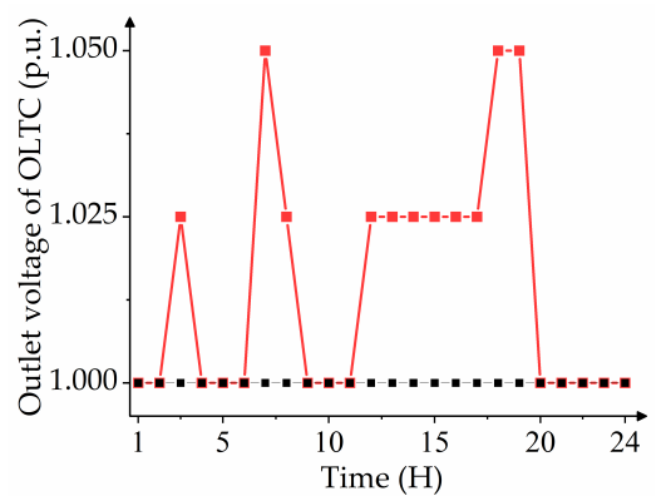

Figure 3. The outlet voltage of an on-load tap changer (OLTC).

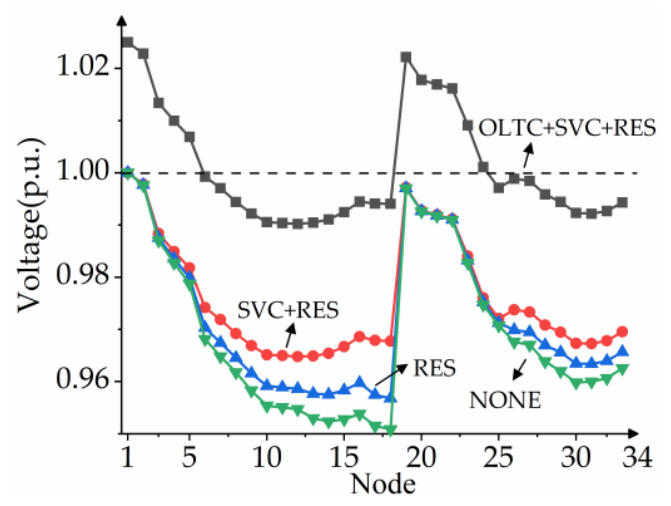

Figure 4. The system voltage distribution at $16 \mathrm{~h}$.

Figure 5 shows the daily load fluctuation ratios of bus 14 and bus 26 in Case 1 and Case 4 at scene 3. From Constraint (7), we can conclude that the load fluctuation caused by the voltage is greater than the reference value if the voltage is greater than $1.00 \mathrm{p} . \mathrm{u}$ and less than the reference otherwise. The symbol $\mathrm{N}$ and $\mathrm{P}$ represent the load change ratios greater than or less than the reference value, respectively. Within $24 \mathrm{~h}$, the average load fluctuation rate of bus 14 was $1.07 \%$ in Case 1 and $0.54 \%$ in Case 4 ; the average load fluctuation rate of bus 26 was $2.45 \%$ in Case 1 and $0.31 \%$ in Case 4 . It was obvious that the rate of load fluctuation in Case 1 was much higher compared with Case 4 . The maximum load fluctuation ratio of bus 14 was $8.02 \%$ at $16 \mathrm{~h}$ in Case 1 and $2.80 \%$ at $19 \mathrm{~h}$ in Case 4 and the biggest voltage deviation of bus 14 was 0.051 p.u. at $16 \mathrm{~h}$ in Case 1 and 0.019 in Case 4, respectively. From the constraint, there was a direct relationship between the load fluctuation with the voltage deviation. If the voltage value is closer to 1.0 p.u., the loss ratio of the bus will be lower. Fo the voltage sensitive loads of bus 26, the maximum loss ratio was $4.08 \%$ at $14 \mathrm{~h}$ in Case 1 and $2.36 \%$ at $20 \mathrm{~h}$ in Case 4 . The corresponding voltage deviation of bus 26 was 0.035 at $14 \mathrm{~h}$ in Case 1 and 0.023 at $20 \mathrm{~h}$ in Case 4. Compared with Case 1, the ratio of the maximum value of voltage deviation in Case 4 was greatly reduced. This demonstrates that Case 4 can not only significantly reduce the loss ratio of highly sensitive loads at different times, but also the overall load loss ratio.

Figure 6 shows the daily power network loss changes of the IEEE-33 bus system. The power network loss in Case 2 was the smallest, and the total power network loss in Case 1 was the largest. The power network loss in Case 4 was slightly higher than that in Case 2. The average power network loss in the four cases were 7.916 in Case 4, 5.396 in Case 3, 13.363 in Case 2, and 19.045 in Case 1.

Table 1 shows the five operating costs in four cases including total cost (TC), day-ahead cost (DAC), real-time cost (RTC), highly sensitive load loss cost (HSLLC), and real-time power network loss cost (RTPNLC). In Case 4, the total cost, day-ahead, and real-time cost were the smallest. In Case 3, the power network loss cost was minimal. However, compared with Case 1, the loss cost of the highly sensitive load of Case 4 was significantly reduced. This paper mainly focused on the loss cost of highly 
sensitive loads. Although the network loss cost produced by Case 4 was not the smallest, compared with other cases, the loss cost of highly sensitive loads was the smallest, which demonstrates that the load fluctuations caused by voltage fluctuations were the smallest. From the point of view that the total cost of the system and the highly sensitive loss cost were the main issues, the adjustment method of Case 4 was the best. Therefore, the coordinated control strategy proposed in this paper can not only significantly reduce the cost of high-sensitivity load loss caused by voltage fluctuations, but also reduce the total power cost and the daily dispatch cost of an ADN.

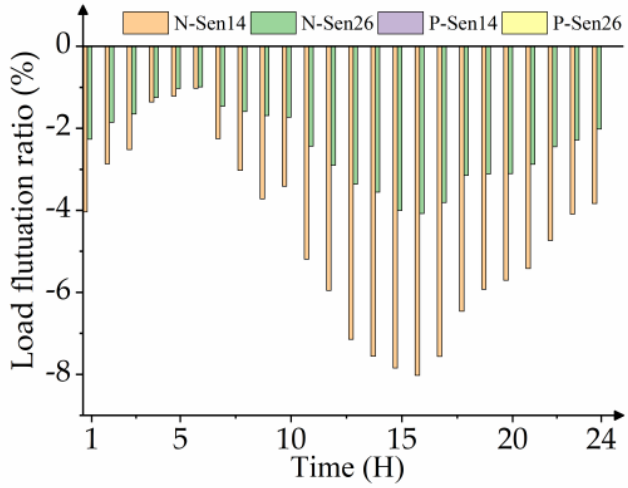

(a) Case 1

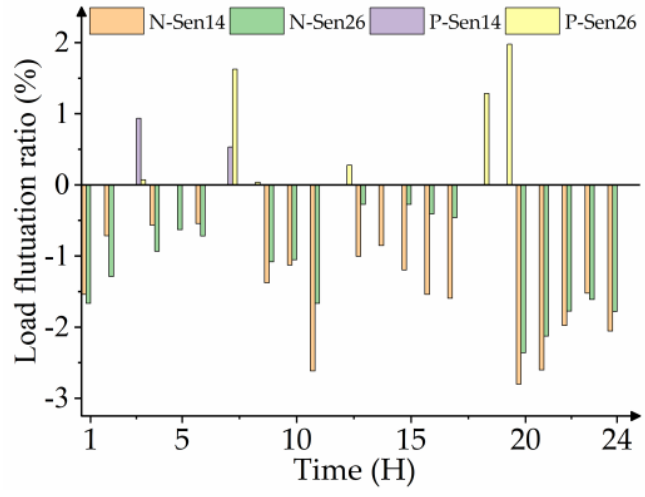

(b) Case 4

Figure 5. The daily load fluctuation ratios of the bus 14 and bus 26 in case 1 (a) and case 4 at scene 3 (b).

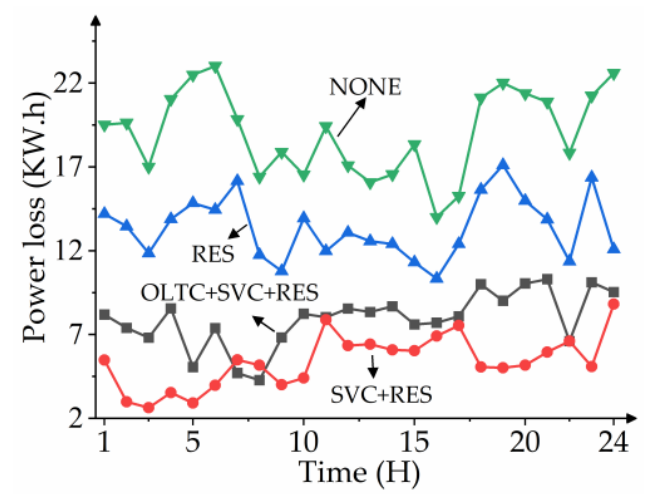

Figure 6. The daily power loss of the ADN in these four cases.

Table 1. Operational cost of the four cases.

\begin{tabular}{ccccc}
\hline Cost (\$) & Cost 1 & Cost $\mathbf{2}$ & Cost $\mathbf{3}$ & Cost 4 \\
\hline TC & 2317.9 & 2263.4 & 2213.9 & 2075.7 \\
DAC & 1970.0 & 1961.3 & 2020.3 & 1972.3 \\
RTC & 347.9 & 302.1 & 193.6 & 103.4 \\
HSLLC & 336.1 & 292.1 & 188.3 & 94.3 \\
RTPNLC & 11.8 & 10 & 5.3 & 9.1 \\
\hline
\end{tabular}

\section{Conclusions}

In order to consider the static voltage characteristics, time characteristics, and uncertainties of highly sensitive loads in scheduling, a linearized voltage sensitive load model with time characteristics was proposed. Furthermore, this paper proposed a linearized two-stage active and reactive power coordination stochastic optimization model to reduce the impact of the uncertainty of load and wind power generation to the sensitive load of these voltages. In the day-ahead optimization, the best OLTC tap and active backup plan was obtained by the active and reactive coordinate strategy to reduce the large voltage fluctuation and providing the sufficient active power reserve to eliminate 
the unbalance power caused by the predict error. In the day-here real-time redispatch, the voltage was further improved by the continuous reactive power compensation device. Finally, our results on the modified IEEE 33-bus system demonstrated the proposed closed-loop coordinate strategy can effectively reduce the loss cost of highly sensitive loads and minimize the economic cost of an ADN by rationally utilizing the reactive power regulation capability of the equipment and the potential of flexible load up- and downregulation.

In this paper, the model of flexible load participating in grid dispatching did not consider the influence of environmental and user willingness factors. However, these factors have an impact on the maximum potential and the response uncertainty of flexible load participation in dispatching. Therefore, how to build a coordinated scheduling model with multiple time scales considering the response uncertainty of a flexible load and the loss cost of highly sensitive loads is our future research direction. Furthermore, the scheduling cost of making a scheduling plan to meet all the uncertainties is very expensive. Therefore, how to introduce the chance constraints in a multiple time scales scheduling model is also our future research.

Author Contributions: Conceptualization, Y.L. and Z.Z.; Methodology, D.S. and Y.L.; Writing-original draft preparation, Y.Z., X.S., Z.Z., and C.Y.; Writing-review and editing, X.S., Z.Z., C.Y., and X.L.; Investigation, Y.Z., Z.Z., and Y.L.; Supervision, Y.L., Z.Z., and D.S.; Funding acquisition, Y.L., Y.Z., and X.S. All authors have read and agreed to the published version of the manuscript.

Funding: This work was supported by the Project of Science and Technology of the State Grid Corporation of China on "Research on Typical Power Supply Mode and Key Technology of Coordinated Operation Control of Distribution System in Highly Sensitive Load Area" (PDB17201800109).

Conflicts of Interest: The authors declare no conflict of interest. The funders had no role in the design of the study; in the collection, analyses, or interpretation of data; in the writing of the manuscript, or in the decision to publish the results.

\section{References}

1. Telukunta, V.; Pradhan, J.; Agrawal, A.; Singh, M.; Srivani, S.G. Protection Challenges under Bulk Penetration of Renewable Energy Resources in Power Systems: A review. CSEE J. Power Energy Syst. 2017, 3, 365-379. [CrossRef]

2. Zheng, W.; Wu, W.; Zhang, B.; Sun, H.; Liu, Y. A Fully Distributed Reactive Power Optimization and Control Method for Active Distribution Networks. IEEE Trans. Smart Grid 2016, 7, 1021-1033. [CrossRef]

3. Aleksey, Z.; Nikita, T.; Denis, S.; Daniil, P.; Vadim, S. A hybrid artificial neural network for voltage security evaluation in a power system. In Proceedings of the 2015 5th International Youth Conference on Energy, Pisa, Italy, 27-30 May 2015. [CrossRef]

4. Feng, P.; Xiao, C.; Guo, S.; Zhang, M.; Li, Y.; Ding, K.; Li, W. Voltage sag vulnerable area identification of a distribution grid with multiple sensitive loads. Power Syst. Prot. Control 2020, 6, 36-44. [CrossRef]

5. Cerbantes, M.C.; Mantovani, J.R.S.; Fernández-Blanco, R.; Ortega-Vazquez, M.A. Optimal Power Flow with Voltage-Sensitive Loads in Distribution Networks. In Proceedings of the IEEE Power and Energy Society General Meeting (PESGM), Boston, MA, USA, 17-21 July 2016. [CrossRef]

6. Ou-Yang, J.-X.; Long, X.-X.; Du, X.; Diao, Y.-B.; Li, M.-Y. Voltage Control Method for Active Distribution Networks Based on Regional Power Coordination. Energies 2019, 12, 4364. [CrossRef]

7. Zheng, Z.; Li, Y.; Xie, X.; Zheng, Y.; Zhang, Z.; Ai, Q. Allocation plan of voltage sags mitigation devices based on life cycle cost. Power Syst. Prot. Control 2018, 9, 128-134. [CrossRef]

8. Le, J.; Zhou, Q.; Wang, C.; Zhao, L. Research on distributed optimal control strategy for a distribution network based on the cooperation of DGs and Var compensators. Power Syst. Prot. Control 2020, 48, 38-47. [CrossRef]

9. Jafari, M.; Naderi, S.B.; Hagh, M.T.; Abapour, M.; Hosseini, S.H. Voltage Sag Compensation of Point of Common Coupling (PCC) Using Fault Current Limiter. IEEE Trans. Power Deliv. 2011, 26, 2638-2646. [CrossRef]

10. Koutsoukis, N.C.; Georgilakis, P.S. A Chance-Constrained Multistage Planning Method for Active Distribution Networks. Energies 2019, 12, 4154. [CrossRef]

11. Cong, P.; Tang, W.; Zhang, L.; Zhang, B.; Cai, Y. Day-Ahead Active Power Scheduling in Active Distribution Network Considering Renewable Energy Generation Forecast Errors. Energies 2017, 10, 1291. [CrossRef] 
12. Chen, F.; Liu, D.; Xiong, X. Research on Stochastic Optimal Operation Strategy of Active Distribution Network Considering Intermittent Energy. Energies 2017, 10, 522. [CrossRef]

13. Hui, H.; Liu, W.; Su, J. Research on multi-objective optimization planning of the active distribution network. In Proceedings of the China International Conference on Electricity Distribution (CICED), Xi'an, China, 10-13 August 2016. [CrossRef]

14. Chen, J.-S.; Xu, T.; Zhou, J.-H.; Wang, X.-X.; Li, T.-C.; Lin, J. Voltage profile optimization of active distribution network through a distributed approach. In Proceedings of the International Conference on Renewable Power Generation (RPG 2015), Beijing, China, 17-18 October 2015. [CrossRef]

15. Martins, V.F.; Borges, C.L.T. Active Distribution Network Integrated Planning Incorporating Distributed Generation and Load Response Uncertainties. IEEE Trans. Power Syst. 2011, 26, 2164-2172. [CrossRef]

16. Xiao, J.X.; Li, Y.; Tan, Y. MILP model for hosting capacity assessment of distributed generation in distribution networks considering ZIP load model. In Proceedings of the IEEE Sustainable Power and Energy Conference (iSPEC), Beijing, China, 21-23 November 2019. [CrossRef]

17. Zhu, J.; Li, Y.; Tan, W. Characteristic Fusion Based on Electric Load Modeling. Power Syst. Technol. 2015, 39, 1358-1364. [CrossRef]

18. Kundur, P. Power System Stability and Control; McGraw Hill: New York, NY, USA, 1994; pp. 377-417.

19. Zhou, H.Z.; Tang, F.D.; Liu, C. Active Distribution Network Dynamic Reconfiguration and DG Dynamic Control Strategy Considering Time-Variant Load. Power Syst. Technol. 2016, 4, 2423-2430. [CrossRef]

20. Wang, Z.Q.; Guo, C.Y.; Liu, W.X.; Li, C.H.; Wang, J.W. Multi-Time-Scale Voltage Control and Coordination Correction of Distribution Network Considering Load Characteristics. Autom. Electr. Power Syst. 2017, $41,51-57$.

21. Ren, J.; Gu, W.; Wang, Y.; Ji, W.; Liu, H.; Cao, G. Multi-time Scale Active and Reactive Power Coordinated Optimal Dispatch in Active Distribution Network Based on Model Predictive Control. Proc. Chin. Soc. Electr. Eng. 2018, 3, 1397-1407. [CrossRef]

22. Lei, Y.; Yang, M.; Han, X.S. A Two-stage Stochastic Optimization of Unit Commitment Considering Wind Power Based on Scenario Analysis. Power Syst. Prot. Control 2012, 40, 58-67.

23. Wang, H.B.; Qi, Y.Z.; Wang, C.G.; Huang, Y.H.; Wang, Y.F. Two-Stage Stochastic Optimal Scheduling Model Considering Flexible Load. Power Syst. Technol. 2018, 42, 3669-3675. [CrossRef]

24. Tian, Z.; Wu, W.; Zhang, B.; Bose, A. Mixed-integer second-order cone programing model for VAR optimization and network reconfiguration in active distribution networks. IET Gener. Transm. Distrib. 2015, 10, 1938-1946. [CrossRef]

25. Li, P.; Li, F.; Song, X.; Zhang, G. Considering the Flexible Load New Energy Access System Optimization for Spinning Reserve. Power Syst. Technol. 2020. [CrossRef]

26. Zheng, N.; Ding, X.-Q.; Guan, Z.-C.; Hu, R.-X.; Miao, H. Coordinated optimization of active power and reactive power in distribution network based on scenario method. Power Syst. Technol. 2019, 43, 1640-1651. [CrossRef]

27. Chen, C.; Wang, F.; Li, C.B.; Liu, P.; Xie, X.H.; Dong, X.Z.; He, R.T. Preventive Reconfiguration of Distribution Networks with Distributed Wind Power. Trans. China Electrotech. Soc. 2013, 29, 172-177. [CrossRef]

28. Yang, Z.; Zhong, H.; Xia, Q.; Kang, C. Solving OPF Using Linear Approximations: Fundamental Analysis and Numerical Demonstration. IET Gener. Transm. Distrib. 2007, 11, 4115-4125. [CrossRef]

29. Yang, J.; Zhang, N.; Kang, C.; Xia, Q. A State-Independent Linear Power Flow Model with Accurate Estimation of Voltage Magnitude. IEEE Trans. Power Syst. 2017, 32, 3607-3617. [CrossRef]

30. Franco, J.F.; Rider, M.J.; Lavorato, M.; Romero, R. A mixed-integer LP model for the optimal allocation of voltage regulators and capacitors in radial distribution systems. Int. J. Electr. Power Energy Syst. 2013, 48, 123-130. [CrossRef]

31. Alguacil, N.; Motto, A.L.; Conejo, A.J. Transmission expansion planning: A mixed-integer LP approach. IEEE Trans. Power Syst. 2003, 18, 1070-1077. [CrossRef]

Publisher's Note: MDPI stays neutral with regard to jurisdictional claims in published maps and institutional affiliations. 